\title{
Nocturnal intermittent hypoxia predicts prevalent hypertension in the European Sleep Apnoea Database cohort study
}

\author{
Ruzena Tkacova ${ }^{1,2}$, Walter T. McNicholas ${ }^{3}$, Martin Javorsky ${ }^{1,2}$, Ingo Fietze ${ }^{4}$, \\ Pawel Sliwinski ${ }^{5}$, Gianfranco Parati ${ }^{6,7}$, Ludger Grote ${ }^{8}$ and Jan Hedner ${ }^{8}$, on behalf \\ of the European Sleep Apnoea Database study collaborators 9
}

\begin{abstract}
Affiliations: 'Dept of Respiratory Medicine, P.J. Safarik University, Medical Faculty, Kosice, Slovakia ${ }^{2}$ L. Pasteur University Hospital, Kosice, Slovakia. ${ }^{3}$ Pulmonary and Sleep Disorders Unit, St Vincent's University Hospital, University College Dublin, Dublin, Ireland. ${ }^{4}$ Schlafmedizinisches Zentrum, Charité, CCM, Berlin, Germany. ${ }^{5}$ Institute of Tubercolosis and Lung Diseases, Dept of Diagnosis and Treatment of Respiratory Failure, Warsaw, Poland. ${ }^{6}$ Dept of Health Sciences, University of Milano-Bicocca, Milan, Italy. ${ }^{7}$ Dept of Cardiology, S. Luca Hospital, Istituto Auxologico Italiano, Milan, Italy. ${ }^{8}$ Dept of Sleep Medicine, Sahlgrenska University Hospital, Gothenburg, Sweden. ${ }^{9}$ For a list of the ESADA study collaborators and their affiliations please see the Acknowledgements section.
\end{abstract}

Correspondence: Walter T. McNicholas, Pulmonary and Sleep Disorders Unit, St Vincent's University Hospital, Elm Park, Dublin 4, Ireland. E-mail: walter.mcnicholasQucd.ie

ABSTRACT Systemic hypertension is associated with obstructive sleep apnoea syndrome (OSAS) but the pathophysiological mechanisms are incompletely understood. A collaborative European network of 24 sleep centres established a European Sleep Apnoea Database to evaluate cardiovascular morbidity associated with OSAS.

11911 adults referred with suspected OSAS between March 2007 and September 2013 underwent overnight sleep studies, either cardiorespiratory polygraphy or polysomnography. We compared the predictive value of the apnoea-hypopnoea index (AHI) and 4\% oxygen desaturation index (ODI) for prevalent hypertension, adjusting for relevant covariates including age, smoking, obesity, dyslipidaemia and diabetes.

Among patients (70\% male, mean \pm SD age $52 \pm 12$ years), $78 \%$ had AHI $>5$ events $\cdot h^{-1}$ and $41 \%$ systemic hypertension. Both AHI and ODI independently related to prevalent hypertension after adjustment for relevant covariates $(\mathrm{p}<0.0001$ for linear trend across quartiles $(\mathrm{Q})$ of severity for both variables). However, in multiple regression analysis with both ODI and AHI in the model, ODI was, whereas AHI was not, independently associated with prevalent hypertension: odds ratios (95\% CI) for Q4 versus Q1 regarding ODI were 2.01 (1.61-2.51) and regarding AHI were $0.92(0.74-1.15)(\mathrm{p}<0.0001$ and $\mathrm{p}=0.3054$, respectively).

This cross sectional study suggests that chronic intermittent hypoxia plays an important role in OSASrelated hypertension.

@ERSpublications

These findings indicate that ODI is superior to AHI in the prediction of hypertension in patients with OSAS http://ow.ly/xFn6R

For editorial comments see page 835 .

This article has supplementary material available from erj.ersjournals.com

Received: Dec 232013 | Accepted after revision: June 042014 | First published online: Aug 072014

Support statement: The authors also acknowledge the financial support of Philips Respironics PLC and ResMed PLC, which each provided unrestricted grants to support overall maintenance of the European Sleep Apnoea Database (ESADA) Project. These companies had no input into the design or implementation of the ESADA Project and played no role in the design or completion of the present study. Nonfinancial support was provided by the European Sleep Research Society and the European Respiratory Society (ERS) in terms of logistics for communication, meetings and data presentations for the ESADA collaborators. The ERS also funded a Task Force to support the evaluation of associations between obstructive sleep apnoea syndrome and hypertension.

Conflict of interest: Disclosures can be found alongside the online version of this article at erj.ersjournals.com 


\section{Introduction}

Obstructive sleep apnoea syndrome (OSAS) affects at least $4 \%$ of adult males and $2 \%$ of adult females in the developed world [1]. OSAS is characterised by repetitive upper airway obstruction during sleep with consequent arterial oxygen desaturation, and apnoeas/hypopnoeas are frequently terminated by microarousals [2]. Cardiovascular diseases represent an important comorbidity and there is evidence that OSAS independently relates to prevalent and incident systemic hypertension in many [3, 4], but not all $[5,6]$ population-based studies. There is also evidence supporting an independent association with ischaemic heart disease, stroke, heart failure, atrial fibrillation and cardiac sudden death [7]. Effective treatment for OSAS with continuous positive airway pressure (CPAP) is associated with considerable benefits in cardiovascular comorbidity, particularly hypertension, both in short-term randomised controlled studies and in long-term observational studies [8-12].

Potential mechanisms of hypertension in OSAS include intermittent hypoxia $[13,14]$, which stimulates systemic inflammation [15], oxidative stress [16] and endothelial dysfunction [17, 18], as well as recurring arousals, which contribute to sympathetic activation [19]. However, the respective contribution of these mechanisms to established hypertension is unclear, and previous population studies of the association of hypertension and OSAS have not addressed this question in detail $[4,20]$.

A collaborative European network of sleep centres was established in 2005, enabled by the European Union Cooperation in Science and Technology Action B26 programme. Within the framework of B26 activities, the European Sleep Apnoea Database (ESADA) project was initiated with the objective to recruit a large prospective international cohort of patients with suspected OSAS. It was argued that a large volume of data collected from a multicentric sleep laboratory population would generate information adding to that obtained in population based cohorts in North America, such as the Wisconsin Sleep Cohort [3] and the Sleep Heart Health Study [4]. In particular, comorbidity and pathophysiological associations may be addressed. Additionally, the cohort also reflects topics such as quality of care, regional differences and influence of local technical routines in patients representing a wide range of European countries.

The present report describes the relationship of OSAS with systemic hypertension in the ESADA cohort and examines the role of various consequences of OSAS in this relationship. The principal objective was to compare measures of recurring intermittent hypoxaemia to measures of sleep disordered breathing and arousal from sleep in the prediction of hypertension.

\section{Methods}

ESADA employs a specifically designed, web-based data collection format constructed for transfer of data from individual centres to the central database at the University of Gothenburg, Sweden. The details of ESADA are described in a separate report, which provides extensive details regarding the study population and the investigative techniques employed [21].

\section{Subjects}

Between March 2007 and September 2013, a cohort of 11911 subjects suspected of having OSAS (8293 males and 3618 females; age 18-80 years, mean \pm SD $52 \pm 12$ years; $78 \%$ with an apnoea-hypopnoea index $(\mathrm{AHI})>5$ events $\cdot \mathrm{h}^{-1}$ ) was assembled in 24 centres across 15 European countries and Israel ( 20 centres were University-affiliated sleep clinics). Three principal exclusion criteria were specified: treatment of OSAS with CPAP or an oral device, severe comorbidity unrelated to OSAS with limited life expectancy, and alcohol or drug abuse within 1 year of inclusion. The appropriate local ethics committee approved the agreed basic protocol.

\section{Demographic characteristics and prevalent hypertension}

Mandatory variables for each subject are reported elsewhere [21]. Current analyses include age, sex, height, weight, neck circumference, waist/hip ratio, smoking history, alcohol intake, details of comorbidities (based on established diagnosis) and antihypertensive medication use. Overweight and obese categories were defined according to WHO classification based on various body mass index (BMI) cut-offs (table 1). Medication use was determined according to the Anatomical Therapeutic Chemical classification system [22]. The method of data collection permitted two levels of confidence in the diagnosis of hypertension, one based on the diagnosis provided by the referring physician without reference to therapy (broad definition) and a second, stricter definition based on those patients diagnosed as hypertensive who were also taking antihypertensive medication (strict definition). Blood pressure measurements taken in the sleep clinic were not used to diagnose hypertension, as these were single readings obtained in the sitting position, which cannot be used as a reliable indicator for a hypertension definition [23]. 
TABLE 1 Distribution of selected characteristics of the European Sleep Apnoea Database study population across apnoeahypopnoea index (AHI) quartiles (Q)

\begin{tabular}{|c|c|c|c|c|c|c|}
\hline & \multirow[t]{2}{*}{ Total } & \multicolumn{4}{|c|}{ AHI quartile } & \multirow[t]{2}{*}{ p-value } \\
\hline & & Q1 & Q2 & Q3 & Q4 & \\
\hline Males & $8293(69.6)$ & $1658(55.7)$ & $1957(66.0)$ & $2250(75.7)$ & $2428(81.0)$ & $<0.0001$ \\
\hline Age years mean \pm SD & $52 \pm 12$ & $47 \pm 13$ & $52 \pm 12$ & $54 \pm 12$ & $53 \pm 12$ & $<0.0001$ \\
\hline Age $\geqslant 60$ years & $3367(28.3)$ & $532(17.9)$ & $853(28.8)$ & $1041(35.0)$ & $941(31.4)$ & $<0.0001$ \\
\hline Former/current & $2888(24.4)$ & $768(25.9)$ & $671(22.8)$ & $679(23.0)$ & $770(26.0)$ & 0.0019 \\
\hline \multicolumn{7}{|l|}{ Body weight } \\
\hline Normal & $1698(14.3)$ & 770 (25.9) & $473(16.0)$ & $321(10.8)$ & $134(4.5)$ & \\
\hline Overweight & 4058 (34.2) & 1174 (39.5) & 1161 (39.3) & $1076(36.3)$ & $647(21.7)$ & \\
\hline Obese & 6119 (51.5) & 1026 (34.5) & $1321(44.7)$ & $1566(52.9)$ & $2206(73.9)$ & $<0.0001$ \\
\hline BMI $\mathrm{kg} \cdot \mathrm{m}^{-2}$ & $31.3 \pm 6.7$ & $28.8 \pm 5.8$ & $30.2 \pm 6.0$ & $31.3 \pm 6.1$ & $34.9 \pm 7.3$ & $<0.0001$ \\
\hline Type 2 diabetes & 1154 (9.7) & $144(4.8)$ & $239(8.1)$ & $325(10.9)$ & $446(14.9)$ & $<0.0001$ \\
\hline Dyslipidaemia & $2481(20.8)$ & $398(13.4)$ & $581(19.6)$ & $720(24.2)$ & $782(26.1)$ & $<0.0001$ \\
\hline COPD & $590(5.0)$ & $137(4.6)$ & $134(4.5)$ & $138(4.6)$ & $181(6.0)$ & 0.0181 \\
\hline Mean $\mathrm{SpO}_{2} \%$ & $93.4 \pm 3.2$ & $94.8 \pm 1.9$ & $94.1 \pm 2.4$ & $93.5 \pm 2.3$ & $91.0 \pm 4.4$ & $<0.0001$ \\
\hline Lowest $\mathrm{SpO}_{2} \%$ & $80.7 \pm 10.9$ & $87.7 \pm 5.8$ & $83.8 \pm 7.5$ & $80.6 \pm 8.0$ & $71.1 \pm 12.9$ & $<0.0001$ \\
\hline Systolic blood pressure $\mathrm{mmHg}$ & $134 \pm 18$ & $130 \pm 18$ & $133 \pm 18$ & $134 \pm 18$ & $137 \pm 18$ & $<0.0001$ \\
\hline Diastolic blood pressure $\mathrm{mmHg}$ & $82 \pm 12$ & $81 \pm 11$ & $82 \pm 11$ & $83 \pm 12$ & $84 \pm 12$ & $<0.0001$ \\
\hline
\end{tabular}

Data are presented as $\mathrm{n}(\%)$ unless otherwise stated. Normal weight defined as having a body mass index (BMI) between 18 and $24.9 \mathrm{~kg} \cdot \mathrm{m}^{-2}$; overweight, between 25 and $29.9 \mathrm{~kg} \cdot \mathrm{m}^{-2}$; and obese, more than $30 \mathrm{~kg} \cdot \mathrm{m}^{-2}$. $\mathrm{p}$-values are for overall F-test or $\chi^{2}$-test. ESS: Epworth Sleepiness Scale; COPD: chronic obstructive pulmonary disease; $\mathrm{SpO}_{2}$ : arterial oxygen saturation measured by pulse oximetry.

\section{Sleep data}

Either polygraphic or polysomnographic (PSG) data were entered in the database, depending on local practice in each sleep centre. All sleep data were manually edited according to protocol definitions before entry. Polygraphic recordings $(n=5910)$ included a minimum of four recording channels (level 3 devices according to the American Academy of Sleep Medicine (AASM)) and, at a minimum, the following variables were recorded: analysed time, subjective sleep time, AHI and oxygen desaturation index (ODI), and mean arterial oxygen saturation measured by pulse oximetry $\left(\mathrm{SpO}_{2}\right)$ and the lowest $\mathrm{SpO}_{2}$ [24]. PSG studies ( $n=6001)$ were performed and analysed according to AASM criteria [25] and analysis comprised the following variables in addition to those indicated for polygraphic recordings: total sleep time; sleep efficiency; percentage of sleep stage 1,2,3 and rapid eye movement (REM) sleep; periodic limb movement (PLM) index; PLM arousal index; respiratory arousal index; spontaneous arousal index; and respiratory disturbance index. Following the 2007 AASM rules, in a polygraphic recording, an apnoea/hypopnoea event was scored if there was a decrease $(\geqslant 50 \%)$ in the amplitude of a valid measure of airflow (either by thermistor or nasal cannula pressure transducer) during sleep (for a hypopnoea, $a \geqslant 3 \%$ oxygen desaturation is required) or the combination of $a \geqslant 30 \%$ reduction in airflow (compared to pre-event baseline) with a $\geqslant 4 \%$ reduction of oxygen saturation. A minimum event duration of $10 \mathrm{~s}$ was required. In a PSG study, in addition to the criteria above, an event (with $\geqslant 50 \%$ flow reduction) associated with arousal was also classified as a hypopnoea. Sleep disordered breathing was assessed with AHI defined as the average number of apnoeas plus hypopnoeas per hour of sleep, and with ODI defined as the number of transient desaturations $(\geqslant 4 \%)$ per hour of sleep for PSG and per hour of analysed time (from lights off to lights on) for polygraphic recordings. The Epworth Sleepiness Scale (ESS) was used to assess daytime sleepiness [26]. Study centres were instructed to state the quality of each polygraphic and PSG recording as "excellent" ( $83.8 \%$ and $78.0 \%$, respectively), "one or two channels missing" ( $9.4 \%$ and $7.8 \%$, respectively), "three or four channels missing" $(0.5 \%$ and $0.1 \%$, respectively), "poor" ( $0.4 \%$ and $0.9 \%$, respectively) and "no information" (5.9\% and $13.2 \%$, respectively). 


\section{Statistical analyses}

The primary sample consisted of 11911 participants; missing data for some covariates reduced the sample size for multivariable models to a minimum of 10949 observations. Using the strict definition of hypertension further reduced the sample size to a minimum of 8533 observations. We examined the association of prevalent hypertension with the two sleep disordered breathing variables of interest, AHI and ODI. We created four equal size groups (quartiles) for AHI and ODI using their percentile distribution in the sample. The lowest quartile $(<25$ th percentile) of the index distribution served as a reference category for the computation of the effect size and associations were quantified by computing relative prevalence odds. All models were adjusted for age, sex, BMI, waist/hip ratio, neck circumference, smoking status, type 2 diabetes, dyslipidaemia, sleep study type, ESS and chronic obstructive pulmonary disease. We refer to these models as "full models". Next, to test the robustness of covariate selection, we used forward stepwise selection procedures to identify parsimonious models of the covariates.

The relative predictive value of AHI versus ODI for prevalent hypertension was evaluated using four approaches. First, we examined individual logistic regression models, where AHI or ODI were entered separately as independent variables. Second, we entered both AHI and ODI simultaneously in logistic regression models, along with the respective covariates. Third, AHI and ODI were introduced into multiple forward stepwise logistic regression analysis together with covariates. Fourth, we examined the additional predictive value of ODI within the groups below and above the median AHI. In testing for the linear trend across the AHI or ODI quartiles in logistic regression models, we entered a single four-level ordinal variable that took the natural logarithm value of the within quartile median. Model fit was tested by HosmerLemeshow goodness of fit test [27]. Age was considered continuously and categorically, as age groups of $18-59$ and $\geqslant 60$ years. The choice of 60 years was arbitrary to assess the role of these factors in an older population sufficiently large for statistical testing. Statistical analyses were conducted with SPSS software version 14.0 (SPSS Inc., Chicago, IL, USA).

\section{Results}

\section{Sample characteristics}

AHI range in the four quartiles was $<6.0,6.0-17.39,17.4-38.9$ and $\geqslant 39.0$ events $\cdot h^{-1}$. For ODI, the quartile ranges were $<3.6,3.6-11.9,12.0-31.69$ and $\geqslant 31.7$ desaturations $\cdot \mathrm{h}^{-1}$. For mean $\mathrm{SpO}_{2}$, the quartile ranges were $<92.2 \%, 92.2-93.9 \%, 94.0-94.9 \%$ and $\geqslant 95.0 \%$. Descriptive characteristics of the study population and the distribution of covariates are presented in table 1 . The study population was strongly dominated by Caucasian ethnicity (93.6\%). Increasing AHI was associated with male sex, higher age, higher obesity indices, and higher prevalence of systemic hypertension, type 2 diabetes and dyslipidaemia.

\section{The effects of $\mathrm{AHI}$ and $\mathrm{ODI}$ on prevalent hypertension in the entire cohort}

Data were analysed for both broad and strict definitions of hypertension. First, we examined individual models (crude or adjusted) where AHI or ODI were entered separately as independent variables. Both AHI and ODI predicted prevalent hypertension for both broad and strict definitions $(\mathrm{p}<0.0001$ for linear trend across ODI and AHI quartiles) (table 2 and online supplementary table 1a). In contrast, in the adjusted (both full and parsimonious) logistic regression models with both AHI and ODI entered simultaneously, only ODI remained a significant predictor of prevalent hypertension using both broad and strict definitions (table 2 and online supplementary table $1 \mathrm{~b}$ ). In the final model of multiple logistic regression analysis, ODI was, while AHI was not, retained as an independent predictor of prevalent hypertension using both definitions (table 3).

Analysis of the predictive value of ODI within the groups categorised by AHI (above and below the median AHI of 17.4 events $\cdot \mathrm{h}^{-1}$ ) revealed that prevalent hypertension increased across increasing quartiles of ODI using both broad and strict definitions ( $\mathrm{p}<0.0001$ for both comparisons) (fig. 1 and online supplementary fig. 1). The type of sleep study (PSG or polygraphy) did not affect the predictive value of AHI or ODI on prevalent hypertension ( $\mathrm{p}$-value for both interaction terms between the type of sleep study and AHI or ODI was >0.05) (broad definition: fig. 2; strict definition: online supplementary table 2).

To increase data robustness, we performed additional analyses on the predictive value of AHI versus mean $\mathrm{SpO}_{2}$ on prevalent hypertension. Similarly to AHI and ODI, mean $\mathrm{SpO}_{2}$ predicted prevalent hypertension for both broad and strict definitions $\left(\mathrm{p}<0.0001\right.$ for linear trend across mean $\mathrm{SpO}_{2}$ quartiles). In the adjusted (both full and parsimonious) logistic regression models with $\mathrm{AHI}$ and mean $\mathrm{SpO}_{2}$ entered simultaneously, $\mathrm{AHI}$ and mean $\mathrm{SpO}_{2}$ remained significant predictors of prevalent hypertension (online supplementary table $3 b)$. 
TABLE 2 Crude and adjusted odds ratios for prevalent systemic hypertension (broad definition) across quartiles (Q) of sleep disordered breathing variables in models containing either apnoea-hypopnoea index (AHI) or oxygen desaturation index (ODI) entered separately or simultaneously as categorical variables

\begin{tabular}{|c|c|c|c|c|c|c|}
\hline Model & Subjects $n$ & Q1 OR & Q2 & Q3 & Q4 & p-value \\
\hline $\mathrm{AHI}$ or ODI entered separately & 10494 & & & & & \\
\hline \multicolumn{7}{|l|}{$\mathrm{AHI}$} \\
\hline Model 0 & 11911 & 1.00 & $2.02(1.81-2.26)$ & $2.82(2.52-3.14)$ & $3.96(3.54-4.42)$ & $<0.0001$ \\
\hline Model 1 & 10949 & 1.00 & $1.29(1.13-1.48)$ & $1.40(1.22-1.60)$ & $1.52(1.31-1.76)$ & $<0.0001$ \\
\hline Model 2 & 11487 & 1.00 & $1.31(1.15-1.49)$ & $1.41(1.23-1.60)$ & $1.51(1.31-1.73)$ & $<0.0001$ \\
\hline \multicolumn{7}{|l|}{ ODI } \\
\hline Model 0 & 11911 & 1.00 & $2.30(2.05-2.58)$ & $3.48(3.11-3.90)$ & $4.86(4.34-5.45)$ & $<0.0001$ \\
\hline Model 1 & 10949 & 1.00 & $1.49(1.30-1.70)$ & $1.73(1.51-1.98)$ & $1.87(1.62-2.17)$ & $<0.0001$ \\
\hline Model 2 & 11487 & 1.00 & $1.48(1.30-1.69)$ & $1.73(1.52-1.98)$ & $1.86(1.62-2.14)$ & $<0.0001$ \\
\hline $\begin{array}{l}\text { Both } \mathrm{AHI} \text { and } \mathrm{ODI} \text { entered } \\
\text { simultaneously }\end{array}$ & 8925 & & & & & \\
\hline \multicolumn{7}{|l|}{$\mathrm{AHI}$} \\
\hline Model 0 & 11911 & 1.00 & $1.27(1.11-1.45)$ & $1.38(1.19-1.60)$ & $1.50(1.25-1.80)$ & $<0.0001$ \\
\hline Model 1 & 10949 & 1.00 & $1.01(0.87-1.17)$ & $0.95(0.79-1.13)$ & $0.92(0.74-1.15)$ & 0.3054 \\
\hline Model 2 & 11487 & 1.00 & $1.02(0.88-1.18)$ & $0.95(0.80-1.13)$ & $0.90(0.73-1.12)$ & 0.2363 \\
\hline \multicolumn{7}{|l|}{ ODI } \\
\hline Model 0 & 11911 & 1.00 & $2.03(1.79-2.31)$ & $2.77(2.38-3.22)$ & $3.56(2.96-4.28)$ & $<0.0001$ \\
\hline Model 1 & 10949 & 1.00 & $1.49(1.28-1.73)$ & $1.79(1.49-2.13)$ & $2.01(1.61-2.51)$ & $<0.0001$ \\
\hline Model 2 & 11487 & 1.00 & $1.48(1.28-1.71)$ & $1.79(1.51-2.13)$ & $2.03(1.64-2.52)$ & $<0.0001$ \\
\hline
\end{tabular}

Data are presented as OR (95\% CI) unless otherwise stated. p-values are for linear trend across quartiles. Model 0: unadjusted; model 1: full model included the covariates age, sex, body mass index, waist/hip ratio, neck circumference, type 2 diabetes, dyslipidaemia, chronic obstructive pulmonary disease, smoking status, Epworth Sleepiness Scale (ESS) score, type of sleep study and sleep disordered breathing variables (i.e. quartiles of $\mathrm{AHI}$ or quartiles of ODI) entered separately or simultaneously into model; model 2: parsimonious model excluded from the full model the covariates waist/hip ratio, ESS score, chronic obstructive pulmonary disease and current smoking status.

\section{Subgroup analysis}

Further analysis in subgroups stratified by age or sex demonstrated similar findings as in the entire cohort; both AHI and ODI predicted prevalent hypertension for both broad and strict definitions but only ODI remained a significant predictor of the outcome variable in both age category groups when AHI and ODI were included together in the statistical analysis. In multiple logistic regression analyses within the respective age categories, ODI was, while AHI was not, retained as an independent predictor of prevalent hypertension (broad definition: online supplementary table 4; strict definition: online supplementary table 5). Analysis of the predictive value of ODI within the groups categorised by AHI (below and above AHI median) in patients $<60$ years revealed similar effect of ODI compared to that seen in the entire cohort: prevalent hypertension increased across increasing quartiles of ODI ( $p$-value for linear trend across ODI quartiles below and above the median AHI: $p=0.0004$ and $p<0.0001$, respectively). A similar analysis in patients $\geqslant 60$ years indicated increases of prevalent hypertension across increasing quartiles of ODI in the subgroup of patients with AHI below but not in the subgroup with AHI above the median AHI value $(\mathrm{p}<0.0001$ and $\mathrm{p}=0.1161$, respectively).

Stratification by sex revealed similar effects of AHI and ODI on prevalent hypertension to those observed in the entire cohort; and in logistic regression analysis stratified by sex, ODI was, while AHI was not, retained as an independent predictor of prevalent hypertension (broad definition: online supplementary table 6; strict definition: online supplementary table 7).

Further, we analysed additional potential confounding factors including study centre for both the broad and strict definitions of hypertension (online supplementary table 8a) and the method for hypopnoea scoring (recommended or alternative criteria according to AASM 2007) for the broad (online supplementary table $8 \mathrm{~b}$ ) and strict (online supplementary table 8c) definition of hypertension. ODI remained the stronger and independent predictor of arterial hypertension in all models.

\section{Discussion}

The present findings confirm previous population-based hallmark studies showing that OSAS is independently associated with prevalent systemic hypertension. While both AHI and ODI were independent predictors of hypertension, ODI was superior to AHI in this prediction. ODI provides a solid reflection of 


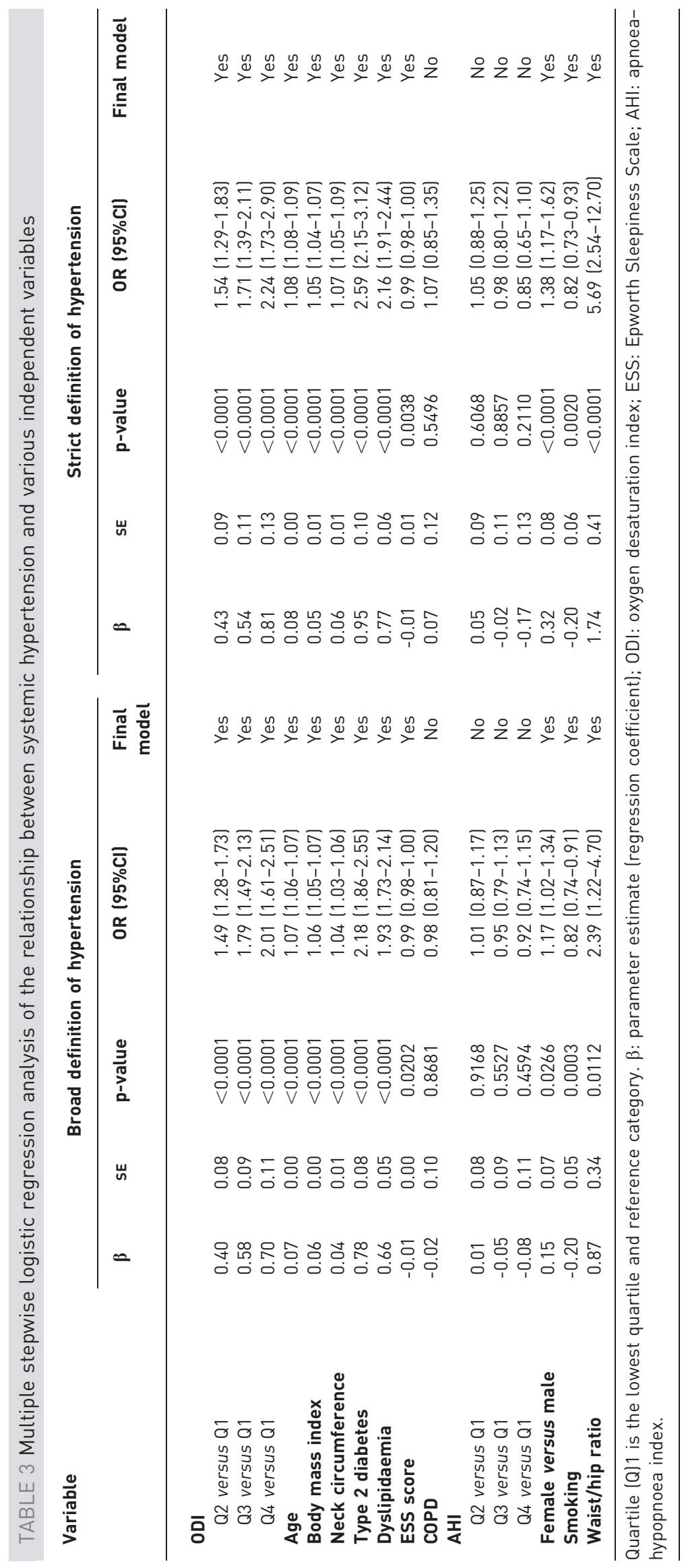




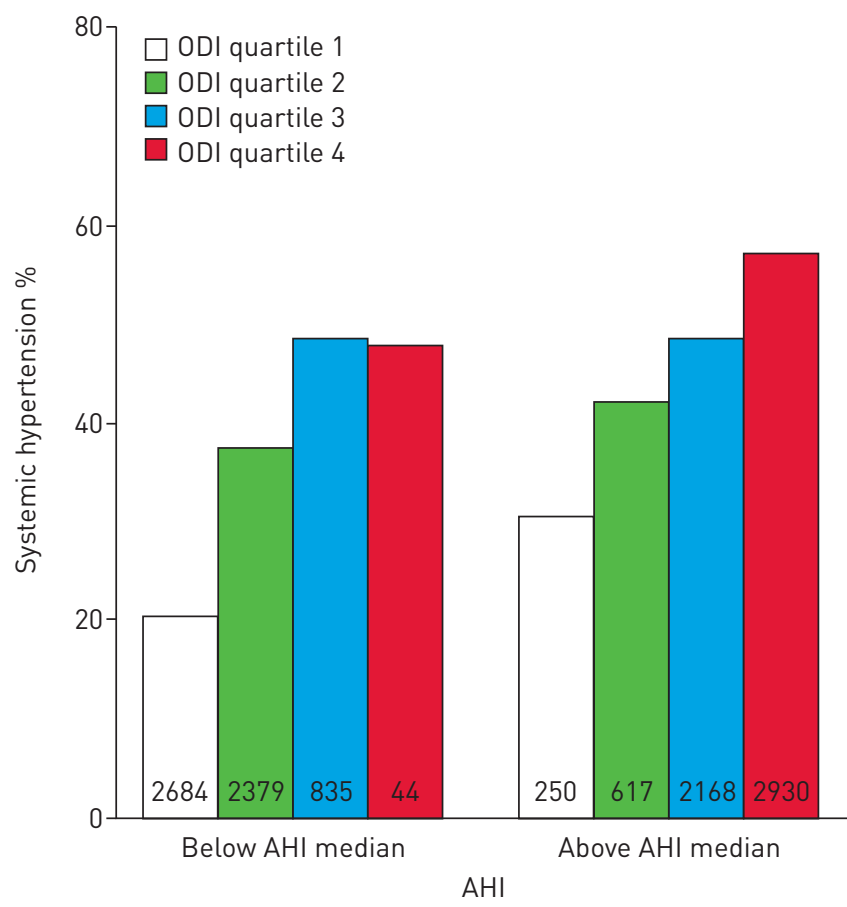

FIGURE 1 Prevalence of systemic hypertension (broad definition) across quartiles of oxygen desaturation index (ODI) within the groups categorised by apnoea-hypopnoea index (AHI) (above and below the median AHI value). Number of subjects per subgroup is shown within bars. $\mathrm{p}<0.0001$ for linear trend across quartiles of ODI for both groups, i.e. below and above the median AHI value.

the degree of intermittent hypoxaemia during sleep. AHI is a more complex measure also reflecting respiratory effort and arousal from sleep, and therefore susceptible to variability in the clinical setting. Our data suggest not only that measures of intermittent hypoxaemia better predict OSA related hypertension but also that intermittent hypoxia is an important mechanism in the pathogenesis of hypertension in OSAS.

Evidence regarding association of hypertension and OSAS

The relationship of hypertension and OSAS is complicated by important confounding variables, particularly obesity. Nonetheless, there is convincing evidence from large population studies that OSAS represents an independent risk factor for systemic hypertension, both in the general population $[3,4,20]$ and in carefully defined cohort studies of sleep clinic patients [28-30]. The Wisconsin Sleep Cohort Study indicated a dose-dependent relationship between AHI at baseline and the incidence of hypertension 4 years later, independent of confounding factors [3]. OSAS is also highly prevalent in patients with systemic hypertension, particularly those with drug-resistant hypertension [31], and the condition is listed as an identifiable cause of hypertension in major hypertension guideline documents [32]. A loss of the normal nocturnal dipping pattern of blood pressure is particularly common in OSAS and may be an early marker of systemic hypertension [33]. Furthermore, prospective randomised trials have shown that effective OSAS treatment decreases blood pressure [8], especially in hypertensive subjects [9].

The present findings provide further evidence supporting an association between OSAS and systemic hypertension, independent of confounding variables such as age, sex, BMI, and relevant comorbidities such as diabetes and dyslipidaemia. They also indicate that the frequency of intermittent hypoxaemia is particularly important in this relationship. Previous reports linking OSAS with hypertension have used AHI as the marker of disease severity and few reports have addressed the contribution of hypoxaemia [4, 34]. In the report from the Sleep Heart Health Study [4] involving over 6000 subjects, both high AHI or sleep time below $90 \%$ oxygen saturation were associated with greater odds of hypertension in a dose-response fashion. However, AHI and ODI were not directly compared in this association. Thus, the present report is the first to detect a specific contribution of intermittent hypoxaemia to prevalent hypertension in a large clinic population.

\section{Mechanisms of hypertension in OSAS}

Obstructive apnoeas/hypopnoeas are typically characterised by systemic hypoxaemia, increased respiratory effort with associated changes in intrathoracic pressure and ultimately microarousal from sleep [35]. These 




FIGURE 2 Multiple logistic regression analysis of the relationship between systemic hypertension (broad definition) and various independent variables according to type of study (polygraphy or polysomnography). Error bars indicate $95 \%$ confidence intervals. Quartile $(\mathrm{Q}) 1$ is the lowest quartile and the reference category. ODI: oxygen desaturation index; AHI: apnoea-hypopnoea index; ESS: Epworth Sleepiness Scale; COPD: chronic obstructive pulmonary disease.

episodes are accompanied by surges in sympathetic nerve activity, with associated increased blood pressure and heart rate. In chronic OSAS, sympathetic drive is persistently elevated, even during wakefulness [36], and muscle sympathetic nerve activity is attenuated during apnoea under hyperoxic conditions [37], supporting a direct link with hypoxaemia. Furthermore, baroreflex sensitivity is diminished in OSAS [35], an effect most closely linked to intermittent hypoxaemia [38]. This derangement of autonomic activity and baroreflex function was attenuated following CPAP treatment [39].

The repetitive hypoxia-reoxygenation associated with obstructive breathing has been suggested to generate systemic inflammation and oxidative stress, which contribute to endothelial dysfunction, vessel wall inflammation and atherosclerosis [16], although this association is still under debate [40]. Thus, by its effects on vascular endothelium, chronic intermittent hypoxaemia may further contribute to impaired blood pressure control both night and day. A causal relationship between intermittent hypoxaemia during sleep and systemic hypertension has been demonstrated in a rat model [41]. Acute intermittent hypoxaemia induces a pressor response in man [13], which is inhibited by angiotensin II receptor blockade [42]. Other proposed mechanisms for hypoxia-induced blood pressure elevation include activation of hypoxiainducible factors [43]. One could also speculate that our data, which demonstrate that ODI may be superior to AHI in the prediction of prevalent hypertension, adds further support to a role for intermittent hypoxaemia in hypertension pathophysiology. Moreover, there may be several pathophysiological explanations including a direct damaging effect on the vascular endothelium as well as arousal-induced induction of increased sympathetic activity. The observational nature of our study does not enable us to determine either the cause or the nature of the better prediction provided by ODI. Finally, ODI may be superior to AHI for methodological reasons, as ODI as a metric has less variance than the more complex and derived AHI.

\section{Study strengths and limitations}

The present data are derived from a large, well-characterised, prospective cohort of patients with clinical features of OSAS [21]. Participating centres were formally evaluated, and agreed to ensure consistent standards of patient recruitment and data acquisition. Study initiation visits were performed at participating sites and a study monitor regularly reviews data quality in the central electronic database. The ESADA study constitutes a relevant clinical setting, recruiting patients from 24 sleep laboratories in 16 countries. Thus, the design mirrors differences in referral pattern and healthcare systems, thereby increasing the generalisability of the present findings. Furthermore, ESADA represents the largest multinational clinical 
cohort study to date exploring associations between OSAS and cardiovascular outcomes, and data derived from a clinic population may provide information with higher clinical validity compared to defined population studies.

The present report has a number of potential limitations. First, the cross-sectional design of this study does not permit us to draw conclusions on a causal relationship between intermittent hypoxaemia and arterial hypertension. Second, data obtained from a sleep clinic population may not be representative of the general population; however, this should not be a significant limitation since the principal objective was to examine relationships between OSAS and hypertension. Third, many patients had limited cardiorespiratory polygraphic sleep studies as opposed to full PSG, which introduces an additional variable in the calculation of AHI and ODI. OSAS severity in sleep studies is traditionally expressed by the AHI, which is the number of apnoeas and hypopnoeas per hour of sleep [44]. However, oxygen desaturation is not a prerequisite for the presence of apnoea or hypopnoea [25], and thus AHI and ODI will probably be different, particularly in PSG studies where arousal forms part of the definition of apnoea and hypopnoea. Therefore, we performed separate analyses for both sleep study types and found comparative results independent of the method used. Fourth, patients were classified as hypertensive based on referring physician diagnosis. Potential diagnostic inaccuracies were limited by performing additional analysis in those hypertensive patients taking antihypertensive medications. This approach was justified because single clinic blood pressure readings were affected by ongoing medication, may be considered to have limited reproducibility and were transiently elevated in some subjects because of a "white coat" effect [45]. Moreover, data in the ESADA reflect the clinical use of different recording systems and scoring routines at various sites across Europe over an extended time period. This practice is likely to introduce increased variability in the assessment of sleep apnoea severity indices. Nevertheless, the robustness of our findings is supported further by extensive confounder analyses addressing type of sleep test, scoring rules applied and recording site. These additional analyses suggest that between-site differences did not systematically influence the association between OSA and hypertension.

\section{Clinical implications}

The present findings confirm the high prevalence of systemic hypertension in OSAS, independent of relevant confounding variables. The finding that the ODI is superior to AHI in the prediction of hypertension is also clinically important, as oxygen saturation is easily measured with high reproducibility in the clinical setting. This may facilitate clinical screening of patients for further differential diagnosis, especially in a cardiology patient population [46].

\section{Acknowledgements}

The European Sleep Apnoea Database study collaborators are F. Barbé (Servei Pneumologia Hospital Arnau de Vilanova and Hospital Santa Maria, Lleida, Spain), M. Bonsignore (Biomedical Department of Internal and Specialistic Medicine (DiBiMIS), University of Palermo, Palermo, Italy), O. Basoglu (Ege University School of Medicine, Izmir, Turkey), P. Escourrou (Service d'Éxplorations Fonctionnelles Multidisciplinaires Hospital Antoine Beclere, Clamart, France), I. Fietze, L. Grote, J. Hedner, J.A. Kvamme (Sleep Laboratory, ENT Dept, Førde Central Hospital, Førde, Norway), P. Lavie (Technion Sleep Laboratory, Rambam Medical Centre, Haifa, Israel), P. Lévy (CHU Grenoble and Université Joseph Fourier, Grenoble, France), C. Lombardi (Instituto Auxologico Italiano, Ospedale San Luca, Milan, Italy), O. Marrone (CNR Institute of Biomedicine and Molecular, Immunology Sleep Laboratory, Palermo, Italy), J.F. Masa (Hospital San Pedro de Alcàntara, Cáceres, Spain), W.T. McNicholas, J.M. Montserrat (Hospital Clinic i Provincial de Barcelona, Barcelona, Spain), G. Nolan (Sleep Disorders Unit, St Vincent's University Hospital, Dublin, Ireland), G. Parati, T. Penzel (Schlafmedizinisches Zentrum, Charite, Berlin, Germany), J.L. Pepin (CHU Grenoble et Université Joseph Fourier, Grenoble, France), M. Pretl (Sleep Disorders Centre, Charles University, Prague, Czech Republic), R. Riha (Dept of Sleep Medicine, Royal Infirmary, Edinburgh, UK), D. Rodenstein (Cliniques universitaires Sant-Luc, Av. Hippocrate, Brussels, Belgium), T. Saaresranta (Turku University Central Hospital Paimio, Hospital Dept of Pulmonary Diseases, Preitilä, Finland), R. Schulz (Sleep Disorders Centre, University of Giessen, Lung Centre, Giessen, Germany), P. Sliwinski, R. Tkacova, G. Varoneckas (Institute Psychophysiology and Rehabilitation, Palanga, Lithuania), J. Verbraecken (Multidisciplinary Sleep Disorders Centre, Antwerp University Hospital and University of Antwerp, Antwerp, Belgium), A. Vitols (Institute of Cardiology, University of Latvia, Riga, Latvia), H. Vrints (Multidisciplinary Sleep Disorders Centre, Antwerp University Hospital and University of Antwerp, Antwerp, Belgium) and J. Zielinski (Dept of Pneumonology, Warsaw Medical University, Warsaw, Poland).

The authors and contributors acknowledge the contribution of the following individuals in data collection: Amna Abdelgabar (Multidisciplinary Sleep Disorders Centre, Antwerp University Hospital and University of Antwerp, Antwerp, Belgium), Zuzana Dorkova (Dept of Respiratory Medicine, P.J. Safarik University, Medical Faculty, Kosice, Slovakia), Lynda Hayes (Multidisciplinary Sleep Disorders Centre, Antwerp University Hospital and University of Antwerp), AnnChristin Lundquist, Christine Mallegho (both Dept of Sleep Medicine, Sahlgrenska University Hospital, Gothenburg, Sweden), Jeanette Norum (Sleep Laboratory, St Vincent's University Hospital, Dublin, Ireland) and Miroslava Pavuckova (L. Pasteur University Hospital, Kosice). 


\section{References}

Young T, Peppard PE, Gottlieb DJ. Epidemiology of obstructive sleep apnea: a population health perspective. Am J Respir Crit Care Med 2002; 165: 1217-1239.

Deegan PC, McNicholas WT. Pathophysiology of obstructive sleep apnoea. Eur Respir J 1995; 8: 1161-1178.

Peppard PE, Young T, Palta M, et al. Prospective study of the association between sleep-disordered breathing and hypertension. N Engl J Med 2000; 342: 1378-1384.

4 Nieto FJ, Young TB, Lind BK, et al. Association of sleep-disordered breathing, sleep apnea, and hypertension in a large community-based study. JAMA 2000; 283: 1829-1836.

5 Cano-Pumarega I, Duran-Cantolla J, Aizpuru F, et al. Obstructive sleep apnea and systemic hypertension: longitudinal study in the general population: the Vitoria Sleep Cohort. Am J Respir Crit Care Med 2011; 184: 1299-1304.

6 O'Connor GT, Caffo B, Newman AB, et al. Prospective study of sleep-disordered breathing and hypertension: the Sleep Heart Health Study. Am J Respir Crit Care Med 2009; 179: 1159-1164.

7 McNicholas WT, Bonsigore MR. Sleep apnoea as an independent risk factor for cardiovascular disease: current evidence, basic mechanisms and research priorities. Eur Respir J 2007; 29: 156-178.

8 Pepperell JC, Ramdassingh-Dow S, Crosthwaite N, et al. Ambulatory blood pressure after therapeutic and subtherapeutic nasal continuous positive airway pressure for obstructive sleep apnoea: a randomised parallel trial. Lancet 2002; 359: 204-210.

9 Becker HF, Jerrentrup A, Ploch T, et al. Effect of nasal continuous positive airway pressure treatment on blood pressure in patients with obstructive sleep apnea. Circulation 2003; 107: 68-73.

10 Marin JM, Carrizo SJ, Vicente E, et al. Long-term cardiovascular outcomes in men with obstructive sleep apnoeahypopnoea with or without treatment with continuous positive airway pressure: an observational study. Lancet 2005; 365: 1046-1053.

11 Doherty LS, Kiely JL, Swan V, et al. Long-term effects of nasal continuous positive airway pressure therapy on cardiovascular outcomes in sleep apnea syndrome. Chest 2005; 127: 2076-2084.

12 Martinez-Garcia MA, Capote F, Campos-Rodriguez F, et al. Effect of CPAP on blood pressure in patients with obstructive sleep apnea and resistant hypertension: the HIPARCO randomized clinical trial. JAMA 2013; 310: 2407-2415.

13 Foster GE, Brugniaux JV, Pialoux V, et al. Cardiovascular and cerebrovascular responses to acute hypoxia following exposure to intermittent hypoxia in healthy humans. J Physiol 2009; 587: 3287-3299.

14 Gilmartin GS, Lynch M, Tamisier R, et al. Chronic intermittent hypoxia in humans during 28 nights results in blood pressure elevation and increased muscle sympathetic nerve activity. Am J Physiol Heart Circ Physiol 2010; 299: H925-H931.

15 Ryan S, Taylor CT, McNicholas WT. Selective activation of inflammatory pathways by intermittent hypoxia in obstructive sleep apnea syndrome. Circulation 2005; 112: 2660-2667.

16 Lavie L. Obstructive sleep apnoea syndrome-an oxidative stress disorder. Sleep Med Rev 2003; 7: 35-51.

17 Carlson JT, Rangemark C, Hedner JA. Attenuated endothelium-dependent vascular relaxation in patients with sleep apnoea. J Hypertens 1996; 14: 577-584.

18 Kohler M, Stoewhas AC, Ayers L, et al. Effects of continuous positive airway pressure therapy withdrawal in patients with obstructive sleep apnea: a randomized controlled trial. Am J Respir Crit Care Med 2011; 184: 1192-1199.

19 Hedner J, Ejnell H, Sellgren J, et al. Is high and fluctuating muscle nerve sympathetic activity in the sleep apnoea syndrome of pathogenetic importance for the development of hypertension? J Hypertens Suppl 1988; 6: S529-S531.

20 Young T, Peppard P, Palta M, et al. Population-based study of sleep-disordered breathing as a risk factor for hypertension. Arch Intern Med 1997; 157: 1746-1752.

21 Hedner J, Grote L, Bonsignore M, et al. The European Sleep Apnoea Database (ESADA): report from 22 European sleep laboratories. Eur Respir J 2011; 38: 635-642.

22 WHO Collaborating Center for Drug Statistics Methodology. Guidelines for ATC classification and DDD assignment. 6th Edn. Oslo, World Health Organization, 2003.

23 Parati G, Stergiou GS, Asmar R, et al. European Society of Hypertension guidelines for blood pressure monitoring at home: a summary report of the Second International Consensus Conference on Home Blood Pressure Monitoring. J Hypertens 2008; 26: 1505-1526.

24 Ferber R, Millman R, Coppola M, et al. Portable recording in the assessment of obstructive sleep apnea. ASDA standards of practice. Sleep 1994; 17: 378-392.

25 Iber C, Ancoli-Israel S, Chesson AL, et al. The AASM Manual for the Scoring of Sleep and Associated Events Rules, Terminology and Technical Specifications. Westchester, American Academy of Sleep Medicine, 2007.

26 Johns MW. A new method for measuring daytime sleepiness: the Epworth sleepiness scale. Sleep 1991; 14: 540-545.

27 Paul P, Pennell ML, Lemeshow S. Standardizing the power of the Hosmer-Lemeshow goodness of fit test in large data sets. Stat Med 2013; 32: 67-80.

28 Grote L, Ploch T, Heitmann J, et al. Sleep-related breathing disorder is an independent risk factor for systemic hypertension. Am J Respir Crit Care Med 1999; 160: 1875-1882.

29 Lavie P, Herer P, Hoffstein V. Obstructive sleep apnoea syndrome as a risk factor for hypertension: population study. BMJ 2000; 320: 479-482.

30 Davies CW, Crosby JH, Mullins RL, et al. Case-control study of 24 hour ambulatory blood pressure in patients with obstructive sleep apnoea and normal matched control subjects. Thorax 2000; 55: 736-740.

31 Logan AG, Perlikowski SM, Mente A, et al. High prevalence of unrecognized sleep apnoea in drug-resistant hypertension. J Hypertens 2001; 19: 2271-2277.

32 Chobanian AV, Bakris GL, Black HR, et al. Seventh report of the Joint National Committee on Prevention, Detection, Evaluation, and Treatment of High Blood Pressure. Hypertension 2003; 42: 1206-1252.

33 Portaluppi F, Provini F, Cortelli P, et al. Undiagnosed sleep-disordered breathing among male nondippers with essential hypertension. J Hypertens 1997; 15: 1227-1233.

34 Roche F, Pepin JL, Achour-Crawford E, et al. At 68 years, unrecognised sleep apnoea is associated with elevated ambulatory blood pressure. Eur Respir J 2012; 40: 649-656.

35 Parati G, Lombardi C, Narkiewicz K. Sleep apnea: epidemiology, pathophysiology, and relation to cardiovascular risk. Am J Physiol Regul Integr Comp Physiol 2007; 293: R1671-R1683. 

sleep apnea. Chest 1993; 103: 1763-1768.

37 Leuenberger U, Jacob E, Sweer L, et al. Surges of muscle sympathetic nerve activity during obstructive apnea are linked to hypoxemia. J Appl Physiol 1995; 79: 581-588.

38 Ryan S, Ward S, Heneghan C, et al. Predictors of decreased spontaneous baroreflex sensitivity in obstructive sleep apnea syndrome. Chest 2007; 131: 1100-1107.

39 Kohler M, Pepperell JC, Casadei B, et al. CPAP and measures of cardiovascular risk in males with OSAS. Eur Respir J 2008; 32: 1488-1496.

40 Kohler M, Ayers L, Pepperell JC, et al. Effects of continuous positive airway pressure on systemic inflammation in patients with moderate to severe obstructive sleep apnoea: a randomised controlled trial. Thorax 2009; 64: 67-73.

41 Fletcher EC. Invited review: Physiological consequences of intermittent hypoxia: systemic blood pressure. J Appl Physiol 2001; 90: 1600-1605.

42 Foster GE, Hanly PJ, Ahmed SB, et al. Intermittent hypoxia increases arterial blood pressure in humans through a renin-angiotensin system-dependent mechanism. Hypertension 2010; 56: 369-377.

43 Prabhakar NR, Semenza GL. Adaptive and maladaptive cardiorespiratory responses to continuous and intermittent hypoxia mediated by hypoxia-inducible factors 1 and 2. Physiol Rev 2012; 92: 967-1003.

44 McNicholas WT. Diagnosis of obstructive sleep apnea in adults. Proc Am Thorac Soc 2008; 5: 154-160.

45 Parati G, Omboni S, Bilo G. Why is out-of-office blood pressure measurement needed? Home blood pressure measurements will increasingly replace ambulatory blood pressure monitoring in the diagnosis and management of hypertension. Hypertension 2009; 54: 181-187.

46 Parati G, Lombardi C, Hedner J, et al. Recommendations for the management of patients with obstructive sleep apnoea and hypertension. Eur Respir J 2013; 41: 523-538. 